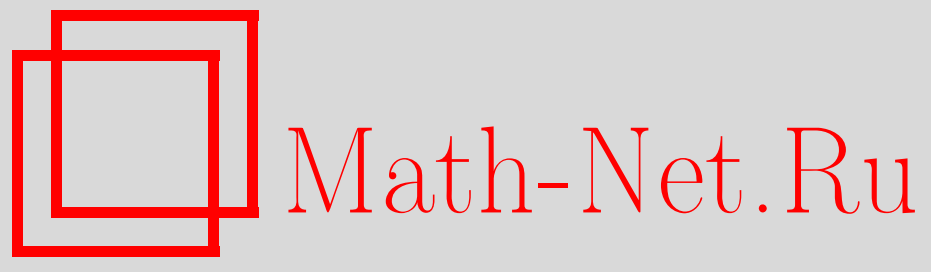

Е. М. Вечтомов, А. В. Черанева, К теории полутел, УМH, 2008, том 63, выпуск 2, 161-162

DOI: https://doi.org/10.4213/rm9069

Использование Общероссийского математического портала Math-Net.Ru подразумевает, что вы прочитали и согласны с пользовательским соглашением http://www . mathnet.ru/rus/agreement

Параметры загрузки:

IP: 3.85 .73 .92

26 апреля 2023 г., $07: 26: 34$

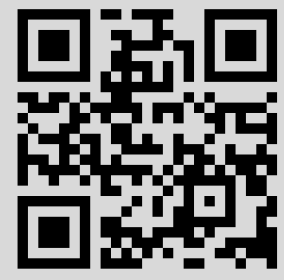




\section{К теории полутел}

\section{Е. М. Вечтомов, А. В. Черанева}

1. Определения. Дистрибутивность и ограниченность. Полутелом называется система, являющаяся одновременно мультипликативной группой и аддитивной коммутативной полугруппой, причем умножение дистрибутивно относительно сложения с обеих сторон. Коммутативное полутело называется полуполем. Всякая конгруэнция на полутеле полностью определяется своим классом единицы, который будем называть ядром. Подмножество $A$ полутела $U$ является ядром тогда и только тогда, когда $A$ - нормальная подгруппа в $U$, для которой выполняется условие: $x, y \in U \& x+y=1 \& a, b \in A \Rightarrow a x+b y \in A[1]$. Ядро конгруэнции, порожденной парой $(1, a)$, будем обозначать $\operatorname{Ker}_{U}(a)$ и называть главным. Решетка $\operatorname{Con} U$ всех конгруэнций полутела $U$ изоморфна решетке его ядер относительно операций пересечения и умножения ядер. Известно, что решетка $\operatorname{Con} U$ всегда модулярна и дистрибутивна для идемпотентных полутел. Полутело $U$ называется: сократимым, если в нем выполняется квазитождество $a+c=b+c \Rightarrow a=b$; идемпотентным, если оно удовлетворяет тождеству $a+a=a$; зероидным, если $a+b=a$ для некоторых его элементов $a$ и $b$; ограниченным, если $U=(2) ;$ дистрибутивным, если решетка $\operatorname{Con} U$ дистрибутивна; простым, если Сon $U$ есть двухэлементная цепь; бирегулярным, если все главные ядра в $U$ дополняемы. Собственное ядро $A$ полутела $U$ называется неприводимым, если из $B \cap C \subseteq A$ следует $B \subseteq A$ или $C \subseteq A$ для любых $B, C \in \operatorname{Con} U$.

Теорема 1 [2]. Для любого полутела U следующие условия эквивалентны:

1) $U$ - ограниченное;

2) $U / A$ - сократимое полутело для любого $A \in \operatorname{Con} U$;

3) решетка Con $U$ изоморфна решетке всех идеалов кольца разностей полутела $U$.

ПредложениЕ 1. Если ядро $K$ полутела $U$ является дистрибутивным полутелом, то и само полутело $U$ дистрибутивно.

ПредлОЖенИЕ 2. Для любого полуполя U решетка конгруэниий его подполуполя (2) есть ретракт решетки $\operatorname{Con} U$.

Теорема 2. Полуполе U дистрибутивно тогда и толъко тогда, когда дистрибутивно его подполуполе (2).

Эти утверждения показывают, что при изучении полутел и полуполей возможно применение методов теории колец.

2. Полутела сечений. Будем обозначать через $\Gamma=\Gamma(X, \Pi)$ полутело сечений пучка П полутел $U_{x}$ над нульмерным компактом $X$ [3]. Компактом называется компактное хаусдорфово пространство. Для произвольной точки $x \in X$ обозначим через $\pi_{x}$ эпиморфизм $\Gamma \rightarrow U_{x}$, определенный правилом $\pi_{x}(s)=s(x)$ для всех $s \in \Gamma$.

Лемма 1. Для любого ядра $A$ полутела $Г$, сечения $a \in A$ и открыто-замкнутого множества $Y \subseteq X$ сечение из $\Gamma$, равное а на $Y$ и 1 на $X \backslash Y$, также принадлежит ядру $A$.

Эта лемма важна в техническом плане; она используется при доказательстве последующих утверждений. Для ее доказательства пучок П расширяется до пучка $\Pi^{\prime}=\Pi \dot{\cup} X$ полутел с нулем $U_{x} \cup\left\{0_{x}\right\}$, в котором $0_{x} \equiv x$ для всех $x \in X$ и $X$ открыто-замкнуто в $\Pi^{\prime}$. Полукольцо сечений $\Gamma\left(X, \Pi^{\prime}\right)$ будет булевым полукольцом [4], множеством обратимых элементов которого служит $\Gamma$.

Лемма 2. Если для ядра $A$ полутела Г верны равенства $\pi_{x}^{-1}(A)=U_{x}$ для всех $x \in X, \operatorname{mo} A=\Gamma$. 
ПредЛОЖениЕ 3. Максимальные (неприводимые) ядра полутела Г - это в точности ядра вида $\pi_{x}^{-1}\left(K_{x}\right)$, где $x \in X u K_{x}$ - максимальное (неприводимое) ядро в $U_{x}$.

ПредЛожениЕ 4. Полутело Г дистрибутивно (ограничено, сократимо, коммутативно, идемпотентно, зероидно) тогда и только тогда, когда дистрибутивны (соответственно: ограничены, сократимы, коммутативны, идемпотентны, зероидны) все слои $U_{x}$.

Теорема 3. Полутело $U=\left(e_{1}\right) \ldots\left(e_{n}\right)$ изоморфно полутелу $\Gamma(X, \Pi)$ сечений некоторого пучка П простых полутел $U_{x}$ над нулвмерным компактом $X$ тогда и только тогда, когда $U$ бирегулярно.

ДоказАтельство. Рассмотрим полутело $\Gamma=\Gamma(X, \Pi)$. Покажем, что главное ядро $A=(a), a \in U$, дополняемо. Рассмотрим открытое множество $Y=\{x \in X \mid$ $\left.a(x)=1_{x}\right\}$ и ядро $B=\{s \in \Gamma \mid s=1$ на $X \backslash Y\}$. Ясно, что $A \cap B=\{1\}$. Если $A B \neq \Gamma$, то по лемме 2 найдется точка $x \in Y$, в которой все сечения из $A B$ равны $1_{x}$. Точка $x$ отделяется от замкнутого множества $X \backslash Y$ некоторым открыто-замкнутым множеством. Значит, по лемме 1 существует сечение $b \in B$, не равное $1_{x}$ в точке $x$; противоречие. Поэтому $A B=\Gamma$, стало быть, $B$ является дополнением $(a)$ в Con $\Gamma$.

Обратно, пусть полутело $U$ бирегулярно. Такое $U$ дистрибутивно. Рассмотрим компактное $T_{1}$-пространство $\mathcal{M}$ всех максимальных ядер полутела $U$ со стоуновской топологией. Поскольку ядро $(u), u \in U$, дополняемо, то, множество $D(u)=\{M \in$ $\mathscr{M} \mid u \notin M\}$ открыто-замкнуто в $\mathcal{M}$. Поэтому $\mathcal{M}$ - нульмерный компакт, и множества $\mathcal{M} \backslash D(u)$ по всем $u \in U$ открыты. Тогда над пространством $\mathcal{M}$ существует пучок П простых полутел $U / M, M \in \mathcal{M}[5]$. Полутело $U$ вкладывается в полутело сечений $\Gamma(\mathcal{M}, \Pi): u \mapsto \hat{u}, \hat{u}(M)=u M \in U / M$ для $u \in U, M \in \mathcal{M}$. Докажем сюръективность `. Пусть $s$ - сечение пучка П. Для любого $M \in \mathcal{M}$ найдется $v \in U$, для которого $\hat{v}=s$ на некоторой открыто-замкнутой окрестности $Y$ точки $M$. Очевидно, ядра $A=\bigcap(\mathcal{M} \backslash Y)$ и $B=\bigcap Y$ являются дополнениями друг друга в $\operatorname{Con} U$. Поэтому найдется элемент $u \in A$, для которого $u^{-1} v \in B$, т. е. $\hat{u}=\hat{v}=s$ на $Y$, $\hat{u}=1$ на $\mathcal{M} \backslash Y$. Эти множества $Y$ образуют открытое покрытие $\mathcal{M}$. Поэтому существует конечное покрытие $\mathcal{M}$ попарно непересекающимися открыто-замкнутыми множествами $Y_{1}, \ldots, Y_{n}$ и существуют такие $u_{1}, \ldots, u_{n} \in U$, что $\hat{u}_{i}=s$ на $Y_{i}$ и $\hat{u}_{i}=1$ на $\mathcal{M} \backslash Y_{i}$ для всех $i=1, \ldots, n$. Тогда $s=\hat{u}_{1} \cdots \hat{u}_{n}=\widehat{u_{1} \cdots u_{n}}$.

СледствиЕ. В условиях теоремы 3 полутело $U$ изоморфно прямому произведению (однозначно определенных) ограниченного полуполя и идемпотентного полутела, а накрывающее пространство П хаусдорфово.

\section{Список литературы}

[1] J. S. Golan, Semirings and their applications, Kluwer, Dordrecht, 1999. [2] А. В. Черанева, Чебъмевский сб., 6:4(16) (2005), 164-171. [3] Г. Е. Бредон, Теория пучков, Наука, М., 1988; пер. с англ.: G. E. Bredon, Sheaf theory, McGraw-Hill Book, New York-Toronto, ON-London, 1967. [4] Е. М. Вечтомов, А. В. Михалёв, В. В. Чермных, Тр. сем. им. И. Г. Петровского, 20 (1997), 282-309; англ. пер.: V. V. Chermnyh, A. V. Mikhalev, E. M. Vechtomov, J. Math. Sci. (N.Y.), 97:3 (1999), 4162-4176. [5] С. В. Полин, Сиб. матем. журн., 15:1 (1974), 90-101; англ. пер. S. V. Polin, "Siberian Math. J.", 15:1 (1974), 64-72.

Е. М. Вечтомов (Е. M. Vechtomov)

Вятский государственный гуманитарный университет E-mail: vecht@mail.ru

А. В. Черанева (А. V. Cheraneva)

Вятский государственный гуманитарный университет

E-mail: A_Cheraneva@inbox.ru
Представлено А. В. Михалёвым Принято редколлегией 17.12.2007 